\title{
ONE-SIDED RESONANCE FOR QUASILINEAR PROBLEMS WITH ASYMMETRIC NONLINEARITIES
}

\author{
KANISHKA PERERA
}

Received 22 October 2001

\section{Introduction}

We consider the quasilinear elliptic boundary value problem,

$$
-\Delta_{p} u=\alpha_{+}(x)\left(u^{+}\right)^{p-1}-\alpha_{-}(x)\left(u^{-}\right)^{p-1}+f(x, u), \quad u \in W_{0}^{1, p}(\Omega),
$$

where $\Omega$ is a bounded domain in $\mathbb{R}^{n}, n \geq 1, \Delta_{p} u=\operatorname{div}\left(|\nabla u|^{p-2} \nabla u\right)$ is the $p$ Laplacian, $1<p<\infty, u^{ \pm}=\max \{ \pm u, 0\}, \alpha_{ \pm} \in L^{\infty}(\Omega)$, and $f$ is a Carathéodory function on $\Omega \times \mathbb{R}$ satisfying a growth condition,

$$
|f(x, t)| \leq q V(x)^{p-q}|t|^{q-1}+W(x)^{p-1},
$$

with $1 \leq q<p$ and $V, W \in L^{p}(\Omega)$. We assume that (1.1) is resonant from one side in the sense that either

$$
\lambda_{l} \leq \alpha_{ \pm}(x) \leq \lambda_{l+1}-\varepsilon
$$

or

$$
\lambda_{l}+\varepsilon \leq \alpha_{ \pm}(x) \leq \lambda_{l+1}
$$

for two consecutive variational eigenvalues, $\lambda_{l}<\lambda_{l+1}$ of $-\Delta_{p}$ on $W_{0}^{1, p}(\Omega)$, and some $\varepsilon>0$ (see Section 2 for the definition of the variational spectrum).

The special case where $\alpha_{+}(x)=\alpha_{-}(x) \equiv \lambda_{l}$ and $q=1$ was recently studied by Arcoya and Orsina [1], Bouchala and Drábek [3], and Drábek and Robinson [8] (see also Cuesta et al. [6] and Dancer and Perera [7]). In the present paper, we prove a single existence theorem for the general case that includes all their results and much more. 
Denote by $N$ the set of nontrivial solutions of the asymptotic problem

$$
-\Delta_{p} u=\alpha_{+}(x)\left(u^{+}\right)^{p-1}-\alpha_{-}(x)\left(u^{-}\right)^{p-1}, \quad u \in W_{0}^{1, p}(\Omega),
$$

and set

$$
F(x, t):=\int_{0}^{t} f(x, s) d s, \quad H(x, t):=p F(x, t)-t f(x, t) .
$$

Our main result is the following theorem.

Theorem 1.1. Problem (1.1) has a solution in the following cases:

(i) equation (1.3) holds and $\int_{\Omega} H\left(x, u_{j}\right) \rightarrow+\infty$,

(ii) equation (1.4) holds and $\int_{\Omega} H\left(x, u_{j}\right) \rightarrow-\infty$ for every sequence $\left(u_{j}\right)$ in $W_{0}^{1, p}(\Omega)$ such that $\left\|u_{j}\right\| \rightarrow \infty$ and $u_{j} /\left\|u_{j}\right\|$ converges to some element of $N$. In particular, (1.1) is solvable when (1.3) or (1.4) holds and $N$ is empty.

As is usually the case in resonance problems, the main difficulty here is the lack of compactness of the associated variational functional, which we will overcome by constructing a sequence of approximating nonresonance problems, finding approximate solutions for them using linking and min-max type arguments, and passing to the limit (see Rabinowitz [10] for standard details of the variational theory). But first we give some corollaries and deduce the results of $[1,3,8]$. In what follows, $\left(u_{j}\right)$ is as in the theorem, that is, $\rho_{j}:=\left\|u_{j}\right\| \rightarrow \infty$ and $v_{j}:=u_{j} / \rho_{j} \rightarrow v \in N$.

First, we give simple pointwise assumptions on $H$ that imply the limits in the theorem.

Corollary 1.2. Problem (1.1) has a solution in the following cases:

(i) equation (1.3) holds, $H(x, t) \rightarrow+\infty$ a.e. as $|t| \rightarrow \infty$, and $H(x, t) \geq-C(x)$,

(ii) equation (1.4) holds, $H(x, t) \rightarrow-\infty$ a.e. as $|t| \rightarrow \infty$, and $H(x, t) \leq C(x)$ for some $C \in L^{1}(\Omega)$.

Note that this corollary makes no reference to $N$.

Proof. If (i) holds, then $H\left(x, u_{j}(x)\right)=H\left(x, \rho_{j} v_{j}(x)\right) \rightarrow+\infty$ for a.e. $x$ such that $v(x) \neq 0$ and $H\left(x, u_{j}(x)\right) \geq-C(x)$, so

$$
\int_{\Omega} H\left(x, u_{j}\right) \geq \int_{v \neq 0} H\left(x, u_{j}\right)-\int_{v=0} C(x) \longrightarrow+\infty
$$

by Fatou's lemma. Similarly, $\int_{\Omega} H\left(x, u_{j}\right) \rightarrow-\infty$ if (ii) holds.

Note that the above argument goes through as long as the limits in (i) and (ii) hold on subsets of $\{x \in \Omega: v(x) \neq 0\}$ with positive measure. Now, taking $w=v^{ \pm}$ in

$$
\int_{\Omega}|\nabla v|^{p-2} \nabla v \cdot \nabla w=\int_{\Omega}\left[\alpha_{+}(x)\left(v^{+}\right)^{p-1}-\alpha_{-}(x)\left(v^{-}\right)^{p-1}\right] w
$$


gives

$$
\begin{aligned}
\left\|v^{ \pm}\right\|^{p} & =\int_{\Omega_{ \pm}} \alpha_{ \pm}(x)\left(v^{ \pm}\right)^{p} \leq\left\|\alpha_{ \pm}\right\|_{\infty}\left\|v^{ \pm}\right\|_{p^{*}}^{p} \mu\left(\Omega_{ \pm}\right)^{p / n} \\
& \leq\left\|\alpha_{ \pm}\right\|_{\infty} S^{-1}\left\|v^{ \pm}\right\|^{p} \mu\left(\Omega_{ \pm}\right)^{p / n}
\end{aligned}
$$

where $\Omega_{ \pm}=\{x \in \Omega: v(x) \gtrless 0\}, p^{*}=n p /(n-p)$ is the critical Sobolev exponent, $S$ is the best constant for the embedding $W_{0}^{1, p}(\Omega) \hookrightarrow L^{p^{*}}(\Omega)$, and $\mu$ is the Lebesgue measure in $\mathbb{R}^{n}$. So

$$
\mu\left(\Omega_{ \pm}\right) \geq\left(S\left\|\alpha_{ \pm}\right\|_{\infty}^{-1}\right)^{n / p}
$$

and hence

$$
\mu(\{x \in \Omega: v(x)=0\}) \leq \mu(\Omega)-S^{n / p}\left(\left\|\alpha_{+}\right\|_{\infty}^{-n / p}+\left\|\alpha_{-}\right\|_{\infty}^{-n / p}\right) .
$$

Thus, we have the following corollary.

Corollary 1.3. Problem (1.1) has a solution in the following cases:

(i) equation (1.3) holds, $H(x, t) \rightarrow+\infty$ in $\Omega^{\prime}$ as $|t| \rightarrow \infty$, and $H(x, t) \geq$ $-C(x)$

(ii) equation (1.4) holds, $H(x, t) \rightarrow-\infty$ in $\Omega^{\prime}$ as $|t| \rightarrow \infty$, and $H(x, t) \leq C(x)$ for some $\Omega^{\prime} \subset \Omega$ with $\mu\left(\Omega^{\prime}\right)>\mu(\Omega)-S^{n / p}\left(\left\|\alpha_{+}\right\|_{\infty}^{-n / p}+\left\|\alpha_{-}\right\|_{\infty}^{-n / p}\right)$ and $C \in$ $L^{1}(\Omega)$.

Similar conditions on $H$ were recently used by Furtado and Silva [9] in the semilinear case $p=2$.

Next, note that

$$
\begin{aligned}
\underline{H}_{+}(x) & \left(v^{+}(x)\right)^{q}+\underline{H}_{-}(x)\left(v^{-}(x)\right)^{q} \\
\leq & \liminf \frac{H\left(x, u_{j}(x)\right)}{\rho_{j}^{q}} \leq \limsup \frac{H\left(x, u_{j}(x)\right)}{\rho_{j}^{q}} \\
\leq & \bar{H}_{+}(x)\left(v^{+}(x)\right)^{q}+\bar{H}_{-}(x)\left(v^{-}(x)\right)^{q},
\end{aligned}
$$

where

$$
\underline{H}_{ \pm}(x)=\liminf _{t \rightarrow \pm \infty} \frac{H(x, t)}{|t|^{q}}, \quad \bar{H}_{ \pm}(x)=\limsup _{t \rightarrow \pm \infty} \frac{H(x, t)}{|t|^{q}} .
$$

Moreover,

$$
\frac{\left|H\left(x, u_{j}(x)\right)\right|}{\rho_{j}^{q}} \leq(p+q) V(x)^{p-q}\left|v_{j}(x)\right|^{q}+\frac{(p+1) W(x)^{p-1}\left|v_{j}(x)\right|}{\rho_{j}^{q-1}}
$$


by (1.2), so it follows that

$$
\begin{aligned}
\int_{\Omega} \underline{H}_{+}\left(v^{+}\right)^{q}+\underline{H}_{-}\left(v^{-}\right)^{q} & \leq \liminf \frac{\int_{\Omega} H\left(x, u_{j}\right)}{\rho_{j}^{q}} \\
& \leq \limsup \frac{\int_{\Omega} H\left(x, u_{j}\right)}{\rho_{j}^{q}} \leq \int_{\Omega} \bar{H}_{+}\left(v^{+}\right)^{q}+\bar{H}_{-}\left(v^{-}\right)^{q} .
\end{aligned}
$$

Thus we have the following corollary.

Corollary 1.4. Problem (1.1) has a solution in the following cases:

(i) equation (1.3) holds and $\int_{\Omega} \underline{H}_{+}\left(v^{+}\right)^{q}+\underline{H}_{-}\left(v^{-}\right)^{q}>0$ for all $v \in N$,

(ii) equation (1.4) holds and $\int_{\Omega} \overline{\bar{H}}_{+}\left(v^{+}\right)^{q}+\overline{\bar{H}}_{-}\left(v^{-}\right)^{q}<0$ for all $v \in N$.

When $\alpha_{+}(x)=\alpha_{-}(x) \equiv \lambda_{1}$ and $q=1$ this reduces to the result of Bouchala and Drábek [3].

Finally, we note that if

$$
\frac{t f(x, t)}{|t|^{q}} \longrightarrow f_{ \pm}(x) \quad \text { a.e. as } t \longrightarrow \pm \infty
$$

then

$$
\frac{F(x, t)}{|t|^{q}}=\frac{1}{|t|^{q}} \int_{0}^{t}\left[\frac{s f(x, s)}{|s|^{q}}-f_{ \pm}(x)\right]|s|^{q-2} s d s+\frac{f_{ \pm}(x)}{q} \longrightarrow \frac{f_{ \pm}(x)}{q}
$$

and hence

$$
\frac{H(x, t)}{|t|^{q}} \longrightarrow\left(\frac{p}{q}-1\right) f_{ \pm}(x)
$$

so Corollary 1.4 implies the following corollary.

Corollary 1.5. Problem (1.1) has a solution in the following cases:

(i) equation (1.3) holds and $\int_{\Omega} f_{+}\left(v^{+}\right)^{q}+f_{-}\left(v^{-}\right)^{q}>0$ for all $v \in N$,

(ii) equation (1.4) holds and $\int_{\Omega} f_{+}\left(v^{+}\right)^{q}+f_{-}\left(v^{-}\right)^{q}<0$ for all $v \in N$.

This was proved in Arcoya and Orsina [1] and Drábek and Robinson [8] for the special case $\alpha_{+}(x)=\alpha_{-}(x) \equiv \lambda_{l}$ and $q=1$.

\section{Proof of Theorem 1.1}

First we recall some facts about the variational spectrum of the $p$-Laplacian. It is easily seen from the Lagrange multiplier rule that the eigenvalues of $-\Delta_{p}$ on $W_{0}^{1, p}(\Omega)$ correspond to the critical values of

$$
J(u)=\int_{\Omega}|\nabla u|^{p}, \quad u \in S:=\left\{u \in W_{0}^{1, p}(\Omega):\|u\|_{p}=1\right\} .
$$


Moreover, J satisfies the Palais-Smale condition (cf. Drábek and Robinson [8]). Thus, we can define an unbounded sequence of min-max eigenvalues by

$$
\lambda_{l}:=\inf _{A \in \mathscr{F}_{l}} \max _{u \in A} J(u), \quad l \in \mathbb{N},
$$

where

$$
\mathscr{F}_{l}=\left\{A \subset S: \exists \text { a continuous odd surjection } h: S^{l-1} \longrightarrow A\right\}
$$

and $S^{l-1}$ is the unit sphere in $\mathbb{R}^{l}$.

LeMma 2.1. $\lambda_{l}$ is an eigenvalue of $-\Delta_{p}$ and $\lambda_{l} \rightarrow \infty$.

Proof. If $\lambda_{l}$ is a regular value of $J$, then there is an $\varepsilon>0$ and an odd homeomorphism $\eta: S \rightarrow S$ such that $\eta\left(J^{\lambda_{l}+\varepsilon}\right) \subset J^{\lambda_{l}-\varepsilon}$ by [2, Theorem 2.5] (the standard first deformation lemma is not sufficient because the manifold $S$ is not of class $C^{1,1}$ when $p<2)$. But then taking $A \in \mathscr{F}_{l}$ with $\max J(A) \leq \lambda_{l}+\mathcal{E}$ and setting $\tilde{A}=\eta(A)$, we get a set in $\mathscr{F}_{l}$ for which $\max J(\tilde{A}) \leq \lambda_{l}-\varepsilon$, contradicting the definition of $\lambda_{l}$. Finally, denoting by $\mu_{l} \rightarrow \infty$ the usual Ljusternik-Schnirelmann eigenvalues, we have $\lambda_{l} \geq \mu_{l}$ since the genus of each $A$ in $\mathscr{F}_{l}$ is $l$, so $\lambda_{l} \rightarrow \infty$.

It is not known whether this is a complete list of eigenvalues when $p \neq 2$ and $n \geq 2$. However, the variational structure provided by this portion of the spectrum is sufficient to show that the associated functional admits a linking geometry in the nonresonant case. We only consider (i) as the proof for (ii) is similar. Let

$$
\alpha_{ \pm}^{j}(x)= \begin{cases}\alpha_{ \pm}(x), & \text { if } \alpha_{ \pm}(x) \geq \lambda_{l}+\frac{1}{j}, \\ \lambda_{l}+\frac{1}{j}, & \text { if } \alpha_{ \pm}(x)<\lambda_{l}+\frac{1}{j},\end{cases}
$$

so that

$$
\lambda_{l}+\frac{1}{j} \leq \alpha_{ \pm}^{j}(x) \leq \lambda_{l+1}-\varepsilon, \quad\left|\alpha_{ \pm}^{j}(x)-\alpha_{ \pm}(x)\right| \leq \frac{1}{j},
$$

and let

$$
\Phi_{j}(u)=\int_{\Omega}|\nabla u|^{p}-\alpha_{+}^{j}(x)\left(u^{+}\right)^{p}-\alpha_{-}^{j}(x)\left(u^{-}\right)^{p}-p F(x, u), \quad u \in W_{0}^{1, p}(\Omega) .
$$

First, we show that there is a $u_{j} \in W_{0}^{1, p}(\Omega)$ such that

$$
\left\|u_{j}\right\|\left\|\Phi_{j}^{\prime}\left(u_{j}\right)\right\| \longrightarrow 0, \quad \inf \Phi_{j}\left(u_{j}\right)>-\infty
$$

By (2.2), there is an $A \in \mathscr{F}_{l}$ such that

$$
J(u) \leq \lambda_{l}+\frac{1}{2 j}, \quad u \in A .
$$


For $u \in A$ and $R>0$,

$$
\begin{aligned}
\Phi_{j}(R u) & =R^{p}\left[J(u)-\int_{\Omega} \alpha_{+}^{j}(x)\left(u^{+}\right)^{p}+\alpha_{-}^{j}(x)\left(u^{-}\right)^{p}\right]-\int_{\Omega} p F(x, R u) \\
& \leq-\frac{R^{p}}{2 j}+p\left(\|V\|_{p}^{p-q} R^{q}+\|W\|_{p}^{p-1} R\right)
\end{aligned}
$$

by (1.2), (2.5), and (2.8), so

$$
\max _{u \in A} \Phi_{j}(R u) \longrightarrow-\infty \quad \text { as } R \longrightarrow \infty
$$

Next, let

$$
\mathscr{S}=\left\{u \in W_{0}^{1, p}(\Omega): \int_{\Omega}|\nabla u|^{p} \geq \lambda_{l+1} \int_{\Omega}|u|^{p}\right\} .
$$

For $u \in \mathscr{Y}$,

$$
\Phi_{j}(u) \geq \varepsilon\|u\|_{p}^{p}-p\left(\|V\|_{p}^{p-q}\|u\|_{p}^{q}+\|W\|_{p}^{p-1}\|u\|_{p}\right)
$$

so

$$
\inf _{u \in \mathscr{S}} \Phi_{j}(u) \geq C:=\min _{r \geq 0}\left[\varepsilon r^{p}-p\left(\|V\|_{p}^{p-q} r^{q}+\|W\|_{p}^{p-1} r\right)\right]>-\infty
$$

Now use (2.10) to fix $R>0$ so large that

$$
\max \Phi_{j}(R A)<C
$$

where $R A=\{R u: u \in A\}$.

Since $A \in \mathscr{F}_{l}$, there is a continuous odd surjection $h: S^{l-1} \rightarrow A$. Let

$$
\Gamma=\left\{\varphi \in C\left(D^{l}, W_{0}^{1, p}(\Omega)\right):\left.\varphi\right|_{S^{l-1}}=R h\right\},
$$

where $D^{l}$ is the unit disk in $\mathbb{R}^{l}$ with boundary $S^{l-1}$. We claim that $R A$ links $\mathscr{S}$ with respect to $\Gamma$, that is,

$$
\varphi\left(D^{l}\right) \cap \mathscr{S} \neq \emptyset \quad \forall \varphi \in \Gamma .
$$

To see this, first note that the proof is done if $0 \in \varphi\left(D^{l}\right)$. Otherwise, denoting by $\pi$ the radial projection onto $S, \tilde{A}:=\pi\left(\varphi\left(D^{l}\right)\right) \cup-\pi\left(\varphi\left(D^{l}\right)\right) \in \mathscr{F}_{l+1}$, and hence

$$
\max _{u \in \pi\left(\varphi\left(D^{l}\right)\right)} J(u)=\max _{u \in \tilde{A}} J(u) \geq \lambda_{l+1}
$$

so $\pi\left(\varphi\left(D^{l}\right)\right) \cap \mathscr{S} \neq \emptyset$, which implies that $\varphi\left(D^{l}\right) \cap \mathscr{S} \neq \emptyset$.

Now it follows from a deformation argument of Cerami [5] that there is a $u_{j}$ such that

$$
\left\|u_{j}\right\|\left\|\Phi_{j}^{\prime}\left(u_{j}\right)\right\| \longrightarrow 0, \quad\left|\Phi_{j}\left(u_{j}\right)-c_{j}\right| \longrightarrow 0
$$


where

$$
c_{j}:=\inf _{\varphi \in \Gamma} \max _{u \in \varphi\left(D^{l}\right)} \Phi_{j}(u) \geq C,
$$

from which (2.7) follows.

We complete the proof by showing that a subsequence of $\left(u_{j}\right)$ converges to a solution of (1.1). It is easy to see that this is the case if $\left(u_{j}\right)$ is bounded, so suppose that $\rho_{j}:=\left\|u_{j}\right\| \rightarrow \infty$. Setting $v_{j}:=u_{j} / \rho_{j}$ and passing to a subsequence, we may assume that $v_{j} \rightarrow v$ weakly in $W_{0}^{1, p}(\Omega)$, strongly in $L^{p}(\Omega)$, and a.e. in $\Omega$. Then

$$
\begin{aligned}
& \int_{\Omega}\left|\nabla v_{j}\right|^{p-2} \nabla v_{j} \cdot \nabla\left(v_{j}-v\right) \\
& =\frac{\left(\Phi_{j}^{\prime}\left(u_{j}\right), v_{j}-v\right)}{p \rho_{j}^{p-1}}+\int_{\Omega}\left[\alpha_{+}^{j}(x)\left(v_{j}^{+}\right)^{p-1}-\alpha_{-}^{j}(x)\left(v_{j}^{-}\right)^{p-1}+\frac{f\left(x, u_{j}\right)}{\rho_{j}^{p-1}}\right]\left(v_{j}-v\right) \longrightarrow 0,
\end{aligned}
$$

and we deduce that $v_{j} \rightarrow v$ strongly in $W_{0}^{1, p}(\Omega)$ (cf. Browder [4]). In particular, $\|v\|=1$, so $v \neq 0$. Moreover, for each $w \in W_{0}^{1, p}(\Omega)$, passing to the limit in

$$
\begin{aligned}
\frac{\left(\Phi_{j}^{\prime}\left(u_{j}\right), w\right)}{p \rho_{j}^{p-1}}= & \int_{\Omega}\left|\nabla v_{j}\right|^{p-2} \nabla v_{j} \cdot \nabla w \\
& -\left[\alpha_{+}^{j}(x)\left(v_{j}^{+}\right)^{p-1}-\alpha_{-}^{j}(x)\left(v_{j}^{-}\right)^{p-1}+\frac{f\left(x, u_{j}\right)}{\rho_{j}^{p-1}}\right] w
\end{aligned}
$$

gives that

$$
\int_{\Omega}|\nabla v|^{p-2} \nabla v \cdot \nabla w-\left[\alpha_{+}(x)\left(v^{+}\right)^{p-1}-\alpha_{-}(x)\left(v^{-}\right)^{p-1}\right] w=0,
$$

so $v \in N$. Thus,

$$
\frac{\left(\Phi_{j}^{\prime}\left(u_{j}\right), u_{j}\right)}{p}-\Phi_{j}\left(u_{j}\right)=\int_{\Omega} H\left(x, u_{j}\right) \longrightarrow+\infty,
$$

contradicting (2.7).

\section{References}

[1] D. Arcoya and L. Orsina, Landesman-Lazer conditions and quasilinear elliptic equations, Nonlinear Anal. 28 (1997), no. 10, 1623-1632.

[2] A. Bonnet, A deformation lemma on a $C^{1}$ manifold, Manuscripta Math. 81 (1993), no. 3-4, 339-359.

[3] J. Bouchala and P. Drábek, Strong resonance for some quasilinear elliptic equations, J. Math. Anal. Appl. 245 (2000), no. 1, 7-19. 


\section{One-sided resonance}

[4] F. E. Browder, Nonlinear eigenvalues problems and group invariance, Functional Analysis and Related Fields (Proc. Conf. for M. Stone, Univ. of Chicago, Illinois, 1968), Springer, New York, 1970, pp. 1-58.

[5] G. Cerami, An existence criterion for the critical points on unbounded manifolds, Istit. Lombardo Accad. Sci. Lett. Rend. A 112 (1978), no. 2, 332-336.

[6] M. Cuesta, D. de Figueiredo, and J.-P. Gossez, The beginning of the Fučík spectrum for the p-Laplacian, J. Differential Equations 159 (1999), no. 1, 212-238.

[7] E. N. Dancer and K. Perera, Some remarks on the Fučík spectrum of the p-Laplacian and critical groups, J. Math. Anal. Appl. 254 (2001), no. 1, 164-177.

[8] P. Drábek and S. B. Robinson, Resonance problems for the p-Laplacian, J. Funct. Anal. 169 (1999), no. 1, 189-200.

[9] M. F. Furtado and E. A. B. Silva, Double resonant problems which are locally nonquadratic at infinity, Proceedings of the USA-Chile Workshop on Nonlinear Analysis (Viña del Mar-Valparaiso, 2000), Southwest Texas State Univ., Texas, 2001, pp. 155-171.

[10] P. H. Rabinowitz, Minimax Methods in Critical Point Theory with Applications to Differential Equations, Published for the Conference Board of the Mathematical Sciences, Washington, DC, 1986.

Kanishra Perera: Department of Mathematical Sciences, Florida Institute of

Technology, 150 West University Boulevard, Melbourne, FL 32901-6975, USA

E-mail address: kperera@winnie.fit.edu

URL: http://winnie.fit.edu/ kperera/ 


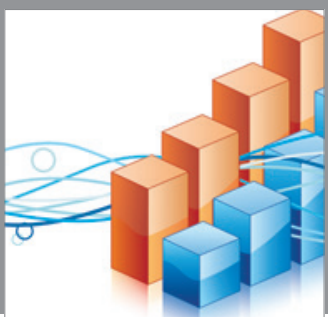

Advances in

Operations Research

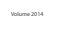

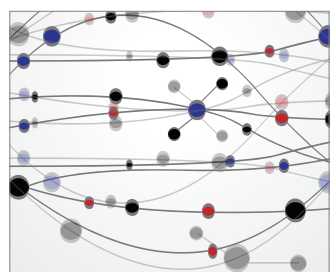

\section{The Scientific} World Journal
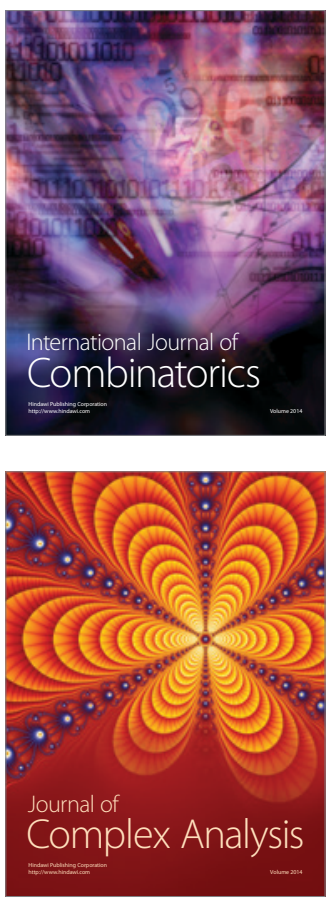

International Journal of

Mathematics and

Mathematical

Sciences
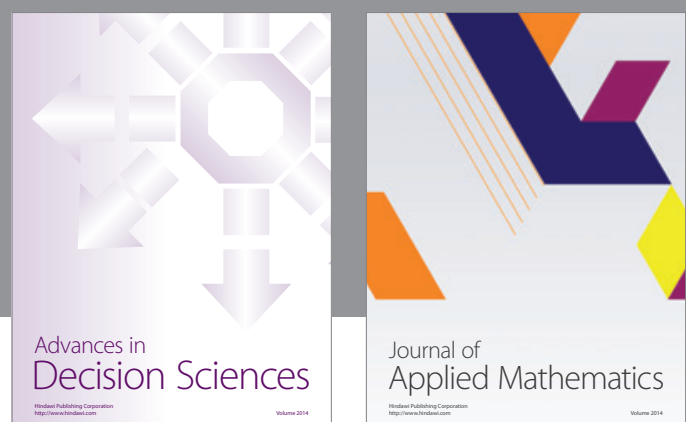

Journal of

Applied Mathematics
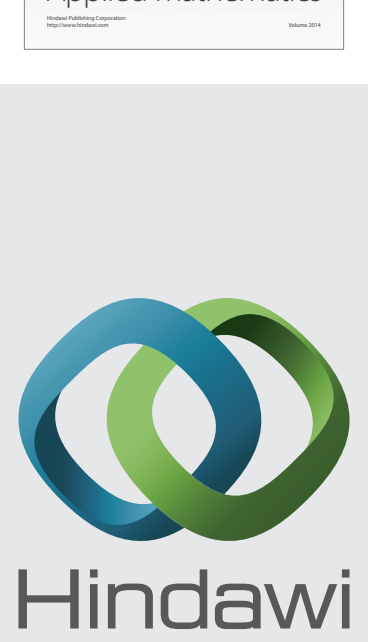

Submit your manuscripts at http://www.hindawi.com
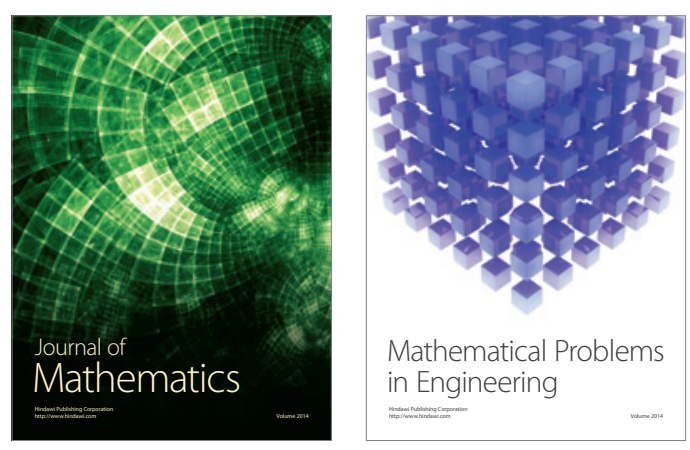

Mathematical Problems in Engineering
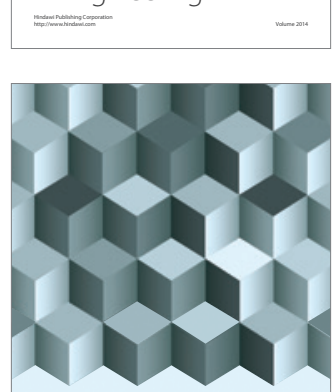

Journal of

Function Spaces
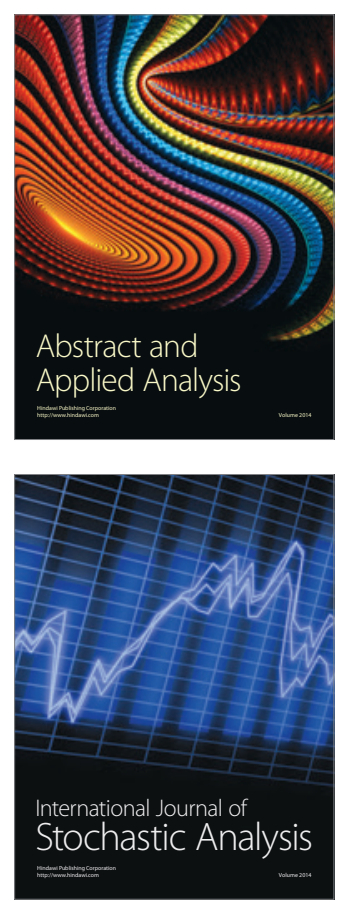

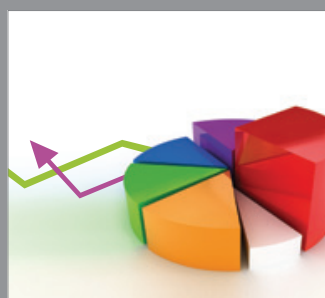

ournal of

Probability and Statistics

Promensencen
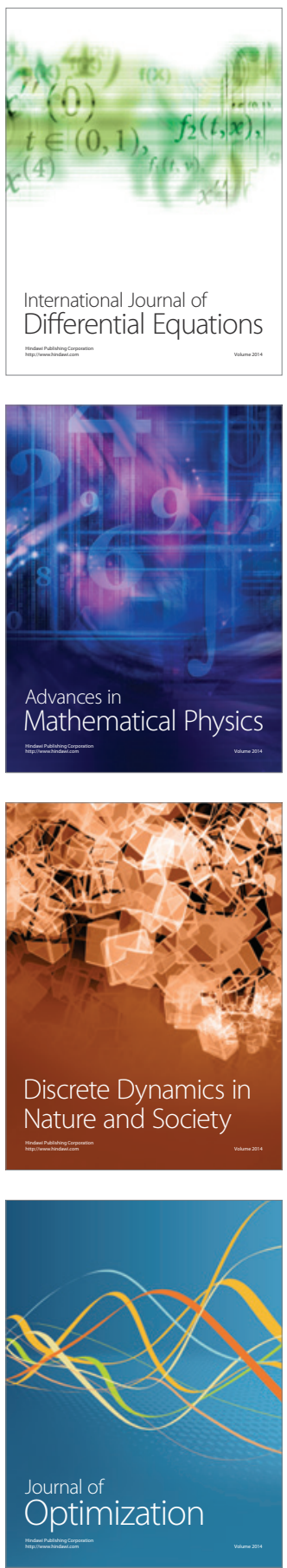